Bayesian Processing for the Detection of Radioactive Contraband from Uncertain Measurements

J. V. Candy, K. Sale, B.L. Guidry, E. Breitfeller, D. Manatt, D. Chambers

June 29, 2007

IEEE CAMSAP '07 
This document was prepared as an account of work sponsored by an agency of the United States government. Neither the United States government nor Lawrence Livermore National Security, LLC, nor any of their employees makes any warranty, expressed or implied, or assumes any legal liability or responsibility for the accuracy, completeness, or usefulness of any information, apparatus, product, or process disclosed, or represents that its use would not infringe privately owned rights. Reference herein to any specific commercial product, process, or service by trade name, trademark, manufacturer, or otherwise does not necessarily constitute or imply its endorsement, recommendation, or favoring by the United States government or Lawrence Livermore National Security, LLC. The views and opinions of authors expressed herein do not necessarily state or reflect those of the United States government or Lawrence Livermore National Security, LLC, and shall not be used for advertising or product endorsement purposes. 


\title{
Bayesian Processing for the Detection of Radioactive Contraband from Uncertain Measurements
}

\author{
James V. Candy, Kenneth Sale, Brian L. Guidry, Eric Breitfeller, Douglas Manatt and David Chambers \\ Lawrence Livermore National Laboratory \\ P.O. 808, L-156 \\ Livermore, CA 94551 \\ Email: candy1@1lnl.gov
}

\begin{abstract}
With the increase in terrorist activities throughout the world, the need to develop techniques capable of detecting radioactive contraband in a timely manner is a critical requirement. The development of Bayesian processors for the detection of contraband stems from the fact that the posterior distribution is clearly multimodal eliminating the usual Gaussian-based processors. The development of a sequential bootstrap processor for this problem is discussed and shown how it is capable of providing an enhanced signal for eventual detection.
\end{abstract}

\section{INTRODUCTION}

The need to investigate new techniques and technologies that can provide for more sensitive detection of terrorist threats around the world demand that meaningful approaches be developed to solving many critical security problems for protection of valuable resources and personnel. With the advent of high power computing, Bayesian techniques have evolved from pure theory and speculation to a viable approach especially in the radiation detection problem. One of the major challenges is to develop techniques that can be applied to time/space dependent problems and provide a timely solution.

Radionuclide detection is a critical technology to detect the transportation of radiological materials by potential terrorists. Detection of these materials is particularly difficult due to the inherent low-count emissions produced. These low-count emissions result when sources are shielded to disguise their existence or, when being transported, are in relative motion with respect to the sensors. Active interrogation with a low intensity neutron source, as required by safety considerations, also produces low-count emissions. The basic problem we propose is to detect, classify and estimate radioactive contraband from highly uncertain (noisy) low-count, radionuclide measurements using a statistical approach based on Bayesian inference and physics-based signal processing. This paper addresses the first step in investigating the problem of enhancing radionuclide signals from noisy radiation measurements using a Bayesian approach. Some work has been accomplished on this problem ([1]-[3]), but unfortunately the physics models incorporated into the processor do not capture the true essence of the problem especially from a signal processing perspective.

Our approach is different in that it models the source radionuclides by decomposing them uniquely as a superposition (union) of monoenergetic sources that are then smeared and distorted as they propagate through the usual path to the output of the detector for measurement and counting. The problems of interest are then defined in terms of this unique representation in which solutions based on extracting this characterization from uncertain detector measurements can be postulated. Using the recently developed particle filters ( $P F)$ suggested by others [4]-[9], and embedding the physics-based models, leads to the formulation of critical problems such as detection, classification and esitmation of threat materials.

We start with the physics-based approach to solving this suite of problems. In the next section we provide background information

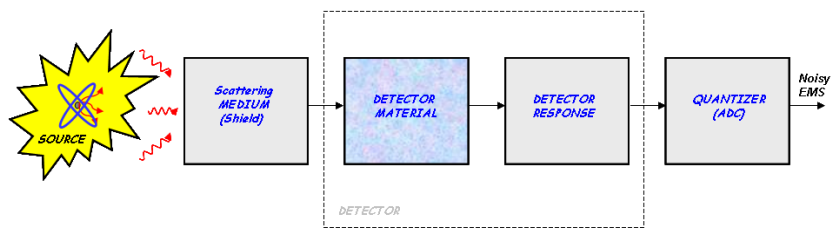

Fig. 1. Gamma-ray evolution and measurement: Radionuclide source (EMS), medium transport (physics), detector material interaction, detector temporal response (preamplification/pulse shaping) and A/D conversion with quantization noise.

on the underlying nuclear process and discuss the monoenergetic model. In section III we discuss the measurement system employed to detect radionuclides and show how the mononenergetic approach leads to a compound Poisson driven Markov process [10] which is amplified, shaped and digitized for further processing. The processor is developed in section IV using state-space representations of the transition probability and associated likelihood. In section V we apply the processor to synthesized data and evaluate its performance. In the final section we summarize our results and discuss future work.

\section{Physics-BASEd Models}

Radiation detection is the unique characterization of a radionuclide based on its electromagnetic emissions. It has been and continues to be an intense area of research and development for well over 50 years ([11]-[13]). It is well-known that a particular radionuclide can be uniquely characterized by two basic properties: its energy emitted in the form of photons or gamma-rays ( $\gamma$-rays) and its radioactive decay rate. Knowledge of one or both of these parameters is a unique representation of a radionuclide. Mathematically, we define the pair, $\left[\left\{\epsilon_{i}\right\},\left\{\lambda_{i}\right\}\right]$, as the respective energy level (MeV) and decay rate (probability of disintegration/nuclei/sec) of the $i^{\text {th }}$-component of the elemental radionuclide. Although either of these parameters can be used to uniquely characterize a radionuclide, only one is actually necessary-unless there is uncertainty in extracting the parameter. Gamma ray spectrometry is a methodology utilized to estimate the energy (probability) distribution or spectrum by creating a histogram of measured arrival data at various levels (count vs. binned energy) [12]. It essentially decomposes the test sample $\gamma$-ray emissions into energy bins discarding the temporal information. The sharp lines are used to identify the corresponding energy bin detecting the presence of a particular component of the radionuclide. In the ideal case, the spectrum consists only of lines or spikes located at the correct bins of each constituent energy, $\epsilon_{i}$, uniquely characterizing the test radionuclide sample.

$\gamma$-ray interactions are subject to the usual physical interaction constraints of scattering and attenuation as well as uncertainties 


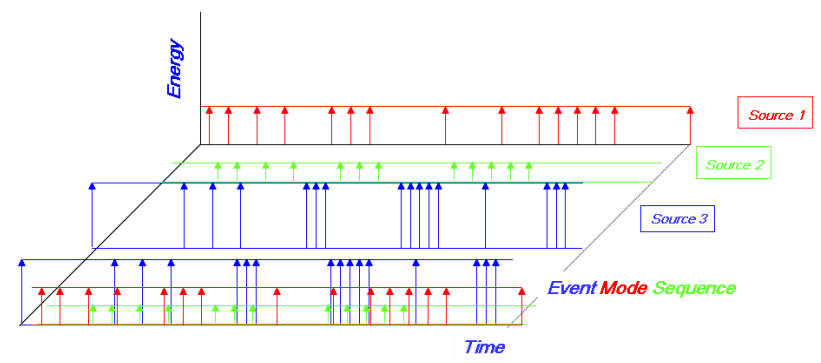

Fig. 2. Monoenergetic Source Decomposition: Individual Constituent EMS and Ideal Composite-free EMS.

intrinsic to the detection process. Energy detectors are designed to estimate the $\gamma$-ray energy from the measured electron current. A typical detector is plagued with a variety of extraneous measurement uncertainty that creates inaccuracy and spreading of the measured current impulse (and therefore $\gamma$-ray energy). The evolution of a $\gamma$-ray as it travels through the medium and interacts with materials, shield and the detector is shown in Fig. 1. It is important to realize that in the diagram, the source radionuclide is represented by its constituents in terms of monoenergetic (single energy level) components and arrival times as $\xi\left(\epsilon_{i}, \tau_{i}\right)$. Since this representation of the source radionuclide contains the constituent energy levels and timing, then all of the information is completely captured by the sets, $\left[\left\{\epsilon_{i}\right\},\left\{\tau_{i}\right\}\right], i=$ $1, \cdots, N_{\epsilon}$. The arrivals can be used to extract the corresponding set of decay constants, $\left\{\lambda_{i}\right\}$ which are related. Thus, from the detector measurement of arrivals, or equivalently the so-called event mode sequence (EMS), a particular radionuclide can be uniquely characterized. The constituent energy levels (spikes), $\left\{\epsilon_{i}\right\}$ and arrival times, $\left\{\tau_{i}\right\}$, extracted from the EMS are depicted in Fig. 2 where we show the union (superposition) of each of the individual constituent monoenergetic sequences composing the complete radionuclide EMS. Note that there is no overlapping of arrivals-a highly improbable event.

So we see that the signal processing model developed from the propagation of the $\gamma$-ray as it travels to the detector is measured and evolves as a distorted EMS. Next, we develop a representation of the event mode sequence in terms of its monoenergetic decomposition. Define $\xi\left(t ; \epsilon_{i}, \tau_{i}, \lambda_{i}\right)$ as the component EMS sequence of the $i^{t h}$ monoenergetic source at time $t$ of energy level (amplitude), $\epsilon_{i}$ and arrival time, $\tau_{i}$ with decay rate, $\lambda_{i}$-as a single impulse, that is, $\xi\left(t ; \epsilon_{i}, \tau_{i}, \lambda_{i}\right)=\epsilon_{i} \delta\left(t-\tau_{i}\right)$ and rate $\lambda_{i}$. Thus, we note that the ideal EMS is composed of sets of energy-time pairs, $\left\{\epsilon_{i}, \tau_{i}\right\}$. In order to define the entire emission sequence over a specified time interval, $\left[t_{o}, T\right)$, we introduce the set notation, $\underline{\tau}_{i}:=\left\{\tau_{i}(1) \cdots \tau_{i}\left(N_{\epsilon}(i)\right)\right\}$ at the $n^{t h}$-arrival with $N_{\epsilon}(i)$ the total number of counts for the $i^{t h}$. source in the interval. Therefore, $\xi\left(t ; \epsilon_{i}, \underline{\tau}_{i}, \lambda_{i}\right)$ results in a unequallyspaced impulse train given by (see Fig. 2 )

$$
\xi\left(t ; \epsilon_{i}, \underline{\tau}_{i}, \lambda_{i}\right)=\sum_{n=1}^{N_{\epsilon}(i)} \xi\left(t ; \epsilon_{i}, \tau_{i}(n), \lambda_{i}\right)=\sum_{n=1}^{N_{\epsilon}(i)} \epsilon_{i} \delta\left(t-\tau_{i}(n)\right)
$$

The interarrival time, is defined by $\triangle \tau_{i}(n)=\tau_{i}(n)-\tau_{i}(n-$ 1) for $\triangle \tau_{i}(0)=t_{o}$ with the corresponding set definition (above) of $\triangle \tau_{i}$ for $i=1, \cdots, N_{\epsilon}(i)-1$.

Extending the EMS model from a single source representation to incorporate a set of $N_{\epsilon}$-monoenergetic sources. Suppose we have a radionuclide source whose EMS is decomposed into its $N_{\epsilon^{-}}$ monoenergetic source components, $\xi(t ; \epsilon, \tau, \lambda)$. From the composi- tion of the EMS we know that

$\xi(t ; \epsilon, \tau, \lambda)=\sum_{i=1}^{N_{\epsilon}} \sum_{n=1}^{N_{\epsilon}(i)} \xi\left(t ; \epsilon_{i}, \tau_{i}(n), \lambda_{i}\right)=\sum_{i=1}^{N_{\epsilon}} \sum_{n=1}^{N_{\epsilon}(i)} \epsilon_{i} \delta\left(t-\tau_{i}(n)\right)$

Clearly, since the EMS is the superposition of Poisson processes, then it is also a composite Poisson process [10] with parameters: $\lambda=\sum_{i=1}^{N_{\epsilon}} \lambda_{i}, \quad \epsilon=\sum_{i=1}^{N_{\epsilon}} \epsilon_{i}, \quad N_{\xi}=\sum_{i=1}^{N_{\epsilon}} N_{\epsilon}(i)$ for $\lambda$ the total decay rate, $\epsilon$ the associated energy levels and $N_{\xi}$ the total counts in the interval, $\left[t_{o}, T\right)$. Note that the composite decay rate is the superposition of all of the individual component rates. This follows directly from the fact that the sum of exponentially (Poisson) distributed variables are exponential (Poisson). We note that the (composite) EMS of the radionuclide directly contains information about $\lambda$, but not about its individual components-unless we can extract the monoenergetic representation (Eq. 2) from the measured data.

Statistically, the EMS can be characterized by the following properties:

- non-uniform arrival time samples, $\tau_{i}(n)$

- monoenergetic source components, $\xi\left(t ; \epsilon_{i}, \tau_{i}(n), \lambda_{i}\right)$ having their own unique decay rate, $\lambda_{i}$

- unique energy level, $\epsilon_{i}$

- gamma distributed arrival times, $\tau_{i}(n), \Gamma\left(k, \tau_{i}\right)$

- Poisson distributed counts, $N_{\epsilon}(i), \mathcal{P}\left(N_{\epsilon}(n)=m\right)$

- exponentially distributed interarrival times, $\triangle \tau_{i}(n)$, $\mathcal{E}\left(\lambda_{i} \triangle \tau_{i}(n)\right)$.

- composite decay rate, $\lambda$

Next we consider the measurement of the EMS along with its inherent uncertainties.

\section{Gamma-Ray Detector Measurements}

Using the mathematical description of the EMS in terms of its monoenergetic source decomposition model discussed previously, we show how this ideal representation must be modified because of the distortion and smearing effects that occur as the $\gamma$-rays propagate according to the transport physics of the radiation process. Typically, these are quantified in terms of $\gamma$-ray spectral properties of energy "peak width" and "peak amplitude". The uncertainties evolve from three factors inherent in the material and instrumentation: inherent statistical spread in the number of charge carriers, variations in the charge collection efficiency and electronic noise [12]. In general, the energy resolution is defined in terms of a Gaussian random variable, $\epsilon_{i} \sim \mathcal{N}\left(\bar{\epsilon}_{i}, \sigma_{\epsilon_{i}}^{2}\right)$

Next we consider uncertainties created in the associated pulse processing system that consists of an amplifier and pulse shaping circuits. Here we concentrate on the amplitude output of the pulse shaper, since it carries not only the quantified $\gamma$-ray energy information, but also it is used for the detector timing circuits (gating pulses, logic pulses, etc.). The shaped pulse is converted to a logic pulse in order to extract precise timing information (arrival times, interarrival times, etc.). We consider the pulse shaper circuitry capable of taking the "raw" material pulse amplifying and shaping it to create a Gaussian pulse shape[12]. Once the Gaussian pulse amplitude, which is proportional to the original $\gamma$-ray energy, is digitized or quantized by the analog-to-digital converter ( $A D C)$, the critical EMS parameters, $\left[\left\{\epsilon_{i}\right\},\left\{\tau_{i}\right\},\left\{\lambda_{i}\right\}\right]$, energy level, arrival time and decay rate can be extracted for further analysis and processing. From this data all other information can be inferred about the identity and quantity of the test radionuclide. 
Next we define a signal processing model that captures the major characteristics of a solid state detector in order to formulate our model-based approach to the radiation detection problem. Consider the diagram again of the overall detector system shown in Fig. 1. Here we see how the EMS is transported through the medium (scattering and attenuation) to the detector and each photon is deposited in the detector material, charge is collected and a voltage created which passes onto pulse shaping electronics that are contaminated with random measurement noise followed by the quantization to produce the noisy output measurement. Thus, from the $i^{t h}$-monoenergetic component we have

$$
\begin{aligned}
p_{m_{i}}(t) & =\sum_{n=1}^{N_{\epsilon}(i)} \xi\left(t ; \epsilon_{i}, \tau_{i}(n), \lambda_{i}\right) \star r(t)+w_{\tau_{i}}(t) \\
& =\sum_{n=1}^{N_{\epsilon}(i)} \epsilon_{i} r\left(t-\tau_{i}(n)\right)+w_{\tau_{i}}(t)
\end{aligned}
$$

where $r(t)$ is a rectangular window of unit amplitude defined within $\tau_{i}(n) \leq t \leq \tau_{i}(n-1)$. The uncertain (random) amplitude is Gaussian, $\epsilon \sim \mathcal{N}\left(\bar{\epsilon}_{i}, \sigma_{\epsilon_{i}}^{2}\right)$, with inherent uncertainty representing the material charge collection process time "jitter" by the additive zeromean, Gaussian noise, $w_{\tau_{i}} \sim \mathcal{N}\left(\bar{\tau}_{i}, \sigma_{w_{\tau_{i}}}^{2}\right)$ and $\underline{\tau}(n) \rightarrow \tau_{i}(n) ; n=$ $1, \cdots, N_{\epsilon}(i)$. Therefore, the material output pulse train for the $i^{t h}$ source is given by $s(t)=H_{S}(t) \star p_{m_{i}}(t)+v(t)$. Extending the model to incorporate all of the $N_{\epsilon}$-sources composing the radionuclide leads to the superposition of all of the monoenergetic pulse trains, that is, $p_{m}(t)=\sum_{i=1}^{N_{\epsilon}} p_{m_{i}}(t)$. The uncertain material pulse, $p_{m}(t)$, is then provided as input to the pulse shaping circuitry. Here the preamplifier and pulse shaper are characterized by a Gaussian filter with impulse response, $H_{S}(t)$ with output given by

$$
s(t)=H_{S}(t) \star p_{m}(t)+v(t)
$$

where the uncertainty created by instrumentation noise is modeled through the additive zero-mean, Gaussian noise source, $v \sim$ $\mathcal{N}\left(0, \sigma_{v}^{2}\right)$. The shaped pulse is then quantized $\left(t_{k} \rightarrow t\right)$ and digitally processed to extract the energy levels and timing information for further processing. Due to quantization limitations the $A D C$ inherently contaminates the measured pulse with zero-mean, Gaussian quantization noise, $v_{q}\left(t_{k}\right)$ while there exists background radiation noise, $b\left(t_{k}\right)$ that must also be taken into account. At this point, we could also develop a signal processing model of the background, but we choose simplicity. We model it as an additive disturbance at the output of the quantizer given by $b\left(t_{k}\right)$ with quantization noise, $v_{q} \sim \mathcal{N}\left(0, \sigma_{q}^{2}\right)$ giving us the final expression as

$$
z\left(t_{k}\right)=s\left(t_{k}\right)+b\left(t_{k}\right)+v_{q}\left(t_{k}\right)
$$

So we see that the entire EMS can be captured in a signal processing model with the key being the monoenergetic source decomposition representation of radiation transport. Next we start with this model and convert it to state-space Markovian form directly for Bayesian processing. In our problem, the EMS is the noisy input sequence characterized by both input and noise processes, that is, $\xi$ and $w_{\tau} \rightarrow w$. The states are part of the preamplifier and Gaussian pulse shaping system and the output is the quantized measurement, that is, $z\left(t_{k}\right) \rightarrow y(t)$. To be more specific, we use $\xi\left(t ; \epsilon_{i}, \tau_{i}, \lambda_{i}\right)$, the $i^{t h}$-monoenergetic source including both amplitude and timing uncertainties as a Poisson input to our Markovian model above along with the matrices, $A, B, C$, specifying the pulse shaping circuit parameters transformed to state-space form, $H_{S} \rightarrow$ Markovian.
To see this consider the state-space representation for a single monoenergetic source is given by the following set of relations:

$$
\begin{array}{rlrc}
\dot{x}_{i}(t) & =A_{i} x_{i}(t)+\mathbf{b}_{i} \xi\left(t ; \epsilon_{i}, \underline{\tau}_{i}, \lambda_{i}\right)+\mathbf{w}_{i} w_{\tau_{i}}(t) & \text { [Source] } \\
y(t) & =\mathbf{c}_{i}^{\prime} x_{i}(t)+v(t) & & \text { [Pulse Shaper] } \\
z\left(t_{k}\right) & =y\left(t_{k}\right)+v_{q}\left(t_{k}\right) ; \quad i=1, \cdots, N_{\epsilon} & \text { [ADC] }
\end{array}
$$

Expanding this model over $i$ to incorporate the $N_{\epsilon^{-}}$ monoenergetic source components gives the extended state vector, $x(t)=\left[x_{i}(t)\left|x_{2}(t)\right| \cdots \mid x_{N_{\epsilon}}(t)\right]^{\prime}$ where each component state is dimensioned $N_{x}$ and therefore, $x \in \mathcal{R}^{N_{x} N_{\epsilon} \times 1}$. Thus, the overall radiation detection state-space model for $N_{\epsilon}$ monoenergetic sources is given by: $A=\operatorname{diag}\left[A_{i}\right], B=\operatorname{diag}\left[B_{i}\right]$, $C=\left[\mathbf{c}_{1}^{\prime}\left|\mathbf{c}_{2}^{\prime}\right| \cdots \mid \mathbf{c}_{N_{\epsilon}}^{\prime}\right]$.

It is interesting to note some of the major properties of this model. The first feature to note is that the monoenergetic decomposition of the radionuclide source is incorporated directly into the model structure. For instance, it we are searching for a particular radionuclide and we know its major energy lines that uniquely describe its spectrum, we can choose the appropriate value of $N_{\epsilon}$ and specify its corresponding mean energy levels and decay rates directly-this is the physics-based approach. We also note that the corresponding noise and statistics are easily captured by this structure as well. This formulation is a continuous-discrete or simply "sampled-data" model, since the $A D C$ in used in the detection scheme.

\section{BAyEsian Physics-BAsed Processor}

In this section we discuss the development of a Bayesian processor for a problem of enhancing a noisy EMS measurement with all of the information required "known" a-priori. We demonstrate how a radiation detector can be modeled (simply) from a physics/statistical signal processing perspective, develop the mathematical representations and incorporate them into a Bayesian framework to enhance the constituent monoenergetic representation. We then demonstrate the Bayesian framework with an illustrative simulation.

A simple radiation transport synthesizer was developed for signal analysis purposes [14]. It consists of specifying the radionuclide in terms of its EMS and corresponding monoenergetic source decomposition then transporting this sequence through the medium (shield) along with its inherent scattering to the detector. At the detector the "surviving" or escaping $\gamma$-ray photons are transported through the detector material (semiconductor) again being absorbed and scattered with the final surviving photons providing the current pulse input to the shaping circuitry as shown in Fig. 1. After initializing the radionuclide and its corresponding monoenergetic source decomposition, the simulator transports the "ideal" EMS through the shield that incorporates both absorption (attenuation) and scattering (Compton) properties using the prescribed shield parameters. The output of this step is specified by the percentage of the photons escaping the shield and those captured or absorbed by the material and converted to thermal energy. The surviving photons escaping are then transported to the detector material where they undergo further absorption and scattering with the survivors converted to charge (electrons) provided as the input to the detector shaping circuitry.

To illustrate the Bayesian approach using physics-based signal processing models, we choose a single monoenergetic source sequence to represent a radionuclide with parameters, $\left\{\epsilon_{o}, \lambda_{o}, N_{\epsilon}(o)\right\}$ and generate the distortion and Gaussian smearing to synthesize the noisy detector output as illustrated previously in Fig. 1. Next we 
investigate the development of a sequential Bayesian processor for the following problem which can be stated formally as: GIVEN a set of noisy $\gamma$-ray detector measurements, $\left\{z\left(t_{k}\right)\right\}$ and a set of a-priori parameters $\left\{\epsilon_{o}, \lambda_{o}, N_{\epsilon}(o)\right\}$ or equivalently its state-space representation, $\Sigma_{o}=\left\{A_{o}, B_{o}, C_{o}\right\}$, along with a known (generated) EMS, $\left\{\xi_{o}(t)\right\}$, FIND best estimate of the underlying radionuclide EMS, $\left\{\hat{y}\left(t_{k}\right)\right\}$.

For our problem we assume we have a good synthetic model of the EMS and we construct the ideal physics-based processor with known parameters $\left\{\epsilon_{o}, \underline{\tau}_{o}, N_{\epsilon}(o)\right\}$ or equivalently known (generated by model) $E M S$. Note that we use the simplified notation, $\xi_{o}(t) \rightarrow$ $\xi\left(t ; \epsilon_{o}, \underline{\tau}_{o}, \lambda_{o}\right)$. Therefore, the state-space representation is given by

$$
\begin{array}{rlrl}
\dot{x}_{o}(t) & =A_{o} x_{o}(t)+b_{o} \xi_{o}(t)+w_{\tau_{o}}(t) & \text { [Process] } \\
y(t) & =c^{\prime} x_{o}(t)+v(t) & & \text { [Measurement] } \\
z\left(t_{k}\right) & =y\left(t_{k}\right)+v_{q}\left(t_{k}\right) & & \text { [ADC] }
\end{array}
$$

where $w_{\tau_{o}} \sim \mathcal{N}\left(0, R_{w_{o} w_{o}}\right), v \sim \mathcal{N}\left(0, R_{v v}\right)$ and $v_{q} \sim \mathcal{N}\left(0, R_{v_{q} v_{q}}\right)$. Under these linear assumptions with additive Gaussian noise processes, the optimal processor is the Kalman filter [15].

In order to develop the particle filter for this problem we require that the transition and likelihood distributions; therefore, under the modeling assumptions (Gaussian noise, known input, parameters, etc.), we have that:

$$
\begin{gathered}
\mathcal{A}(x(t) \mid x(t-1)) \sim \mathcal{N}\left(A_{o} x(t-1)+B_{o} \xi_{o}(t), R_{w_{\tau o} w_{\tau o}}(t-1)\right) \\
\mathcal{C}(y(t) \mid x(t)) \sim \mathcal{N}\left(C_{o} x(t-1), R_{v v}\right)
\end{gathered}
$$

Therefore, the bootstrap particle filter implementation for this problem for $i=1, \cdots, N_{p}$ is:

- Draw: $x_{i}(t) \sim \mathcal{A}\left(x(t) \mid x_{i}(t-1)\right) ; w_{i} \sim \operatorname{Pr}\left(w_{i}(t)\right)$;

- Weight: $W_{i}(t)=\mathcal{C}(y(t) \mid x(t))$;

- Normalize: $\mathcal{W}_{i}(t)=W_{i}(t) / \sum_{i=1}^{N_{p}} W_{i}(t)$;

- Resample: $\hat{x}_{i}(t) \Rightarrow x_{i}(t)$;

- Posterior: $\hat{\operatorname{Pr}}\left(x(t) \mid Y_{t}\right) \approx \sum_{i} \mathcal{W}_{i}(t) \delta\left(x(t)-\hat{x}_{i}(t)\right)$;

- Inferences: $\hat{x}(t \mid t), \hat{x}_{M A P}(t)$.

This completes the formulation and Bayesian processor realizations both for the Kalman and particle filter designs, next we synthesize a radiation detection problem and apply the processors.

Suppose we have a nuclide represented by a single monoenergetic source of energy level, $\epsilon_{o}=3.086 \mathrm{keV}$. Using the transport simulator with the following Gaussian noise variances: $R_{w w}=10^{-6}$ and $R_{v v}=10^{-2}$, we generated a realization of the noisy EMSNंext we construct the EMS signal enhancer and the results are shown in Fig. 3 where we observe the raw synthesized data illustrated along with the enhanced Bayesian processor estimates (both conditional mean and maximum a-posteriori). We see the enhanced EMS signal in (a) along with a zoomed version to observe the actual enhancement. Note the zero amplitude level noise has been minimized as part of the enhancement process. The optimal, $X_{o p t}$ (Kalman filter), and particle filter inferences for both conditional mean and maximum a-posteriori are annotated in Fig. 3; however, all of the realizations overlay one another so they are hard to differentiate. This completes the section, next we summarize our results and discuss future work.

\section{SUMmarY}

We have demonstrated the development of Bayesian processing for the radiation detection problem and shown how the physics-based signal processing models evolve using the EMS as the foundation for this approach. The detection process evolved from the physics directly and was incorporated into a Markovian (state-space) representation enabling the development of the sequential Bayesian paridigm.
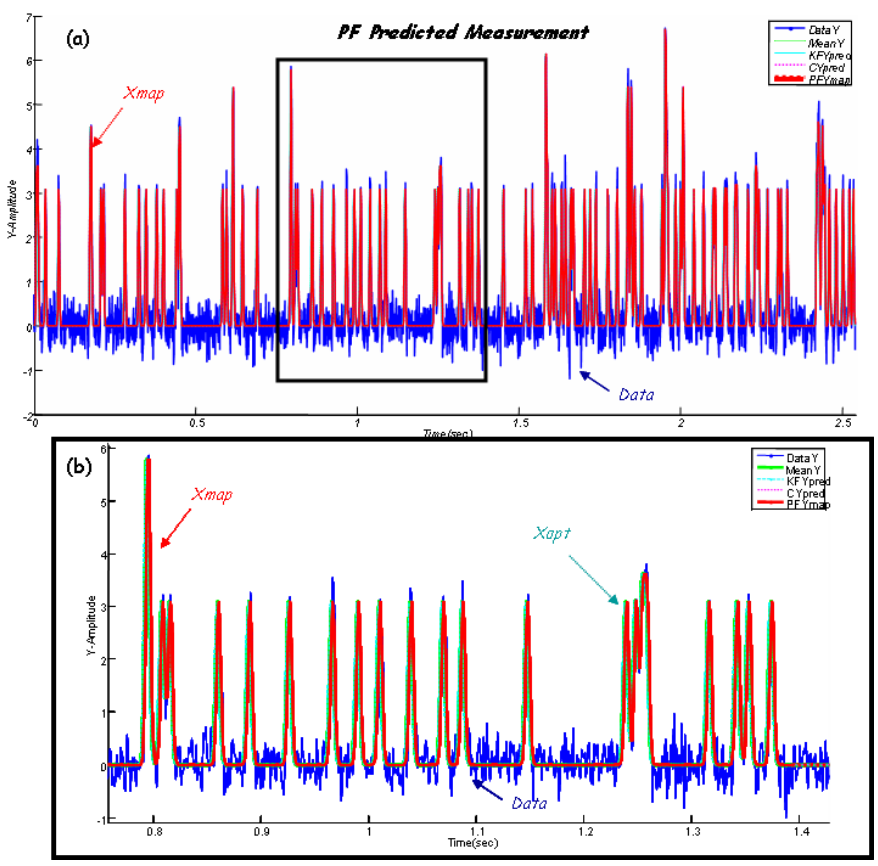

Fig. 3. Bayesian Processor for Radiation Detection Signal Enhancement. (a) Entire EMS enhancement with box annotating zoom area. (b) Zoomed EMS with raw and enhanced processor outputs.

\section{REFERENCES}

[1] C. Andrieu, E. Barat and A. Doucet, "Bayesian deconvolution of noisy filtered point processes, IEEE Trans. Sig. Proc., Vol. 49, 2001.

[2] A. Doucet and P. Duvaut, "Bayesian estimation of state space models applied to deconvolution of Bernoulli-Gaussian processes, Signal Process., Vol. 57, 1997.

[3] S. Gulam Razul, W. J. Fitzgerald and C. Andrieu, "Bayesian model selection and parameter estimation of nuclear emission spectra using RJMCMC, Nucl. Instrum. And Methods in Physics Res. A, Vol. 497, 2003.

[4] A. Doucet, N. de Freitas and N. Gordon, Sequential Monte Carlo Methods in Practice, Springer-Verlag: New York, 2001.

[5] J. Liu, Monte Carlo Strategies in Scientific Computing, Springer-Verlag: New York, 2001.

[6] B. Ristic B., S. Arulampalam and N. Gordon, Beyond the Kalman Filter: Particle Filters for Tracking Applications, Boston: Artech House, 2004

[7] S. Godsill and P. Djuric, "Special Issue: Monte Carlo methods for statistical signal processing." IEEE Trans. Signal Proc., vol. 50, 2002.

[8] P. Djuric, J. Kotecha, J. Zhang, Y. Huang, T. Ghirmai, M. Bugallo and J. Miguez, "Particle Filtering." IEEE Signal Proc. Mag. vol. 20, No. 5, pp. 19-38, 2003.

[9] A. Doucet and X. Wang, "Monte Carlo methods for signal processing," IEEE Signal Proc. Mag. vol. 24, No. 5, pp. 152-170, 2005.

[10] D. L. Snyder and M. I. Miller, Random Point Process in Time and Space. (New York: Springer-Verlag, 1991).

[11] R. D. Evans, The Atomic Nucleus. (New York, N.Y.: McGraw-Hill, 1985).

[12] G.F. Knoll, Radiation Detection and Measurement, 3rd Ed., (Hoboken N.J.: John Wiley, 2000).

[13] L. J. Meng and D. Ramsden, "An inter-comparison of three spectraldeconvolution algorithms for gamma-ray spectroscopy, IEEE Trans. Nucl. Sci., Vol. 47, 4, 2000.

[14] A. Meyer, M. Axelrod, J. Candy, K. Sale, D. Slaughter and S. Putkin, "Systematic approach to gamma ray detection," LLNL Report, (in progress), 2006.

[15] J. V. Candy, Model-Based Signal Processing, John Wiley/IEEE Press: New Jersey, 2006.

This work was performed under the auspices, of the $U$. S. DOE by UC, LLNL under Contract W-7405-Eng-48. 